\title{
DU MAUVAIS USAGE DE LA TRADITION RECONSTITUÉE : LA MÉDIATION EXTRAJUDICIAIRE EN CHINE ${ }^{1}$
}

\section{Hélène Piquet*}

L'étude porte sur le renouveau de la médiation extrajudiciaire en Chine abordé sous l'angle de l'accès à la justice. Ce renouveau est situé dans son contexte politique, notamment dans l'appel aux paradigmes locaux lui servant de toile de fond. Le régime de la Loi sur la médiation populaire de République Populaire de Chine, en vigueur depuis le $1^{\text {er }}$ janvier 2011, est présenté pour ensuite faire place à une étude des problèmes de mise en cuvre de la loi.

The study analyses the renewal of extrajudicial mediation in China in terms of access to justice. This renewal is discussed in its political context, notably the fact that the legislation resorts to local paradigms and uses them as background for the process. After having described the system established by the People's Republic of China's People's Mediation Law, which has come into force on January 1, 2011, the document examines the problems raised by the implementation of the Law.

\section{INTRODUCTION}

La médiation extrajudiciaire, conçue comme un nouveau mode de règlement des conflits, se définit comme un processus par lequel un tiers impartial facilite les négociations entre deux ou plusieurs parties en conflit, en fondant ce processus sur les intérêts et les besoins de ces mêmes parties ${ }^{2}$. Nous adoptons la terminologie de Nadja Alexander, qui parle de médiation moderne. Il s'agit ici de la médiation extrajudiciaire telle qu'elle a été élaborée dans la décennie 1970 aux États-Unis, puis, plus tard, en Australie, au Royaume-Uni, dans les pays européens de droit civil et enfin en Afrique du Sud ${ }^{3}$. Elle a suscité de nombreuses critiques lors de son essor aux États-Unis et en Europe comme en témoignent, entre autres, les travaux d'Owen Fiss, de Laura Nader et de Marc Galanter'. Ces travaux étant très connus,

1 Texte publié grâce à une subvention du CRSH. L'auteur tient à remercier le comité éditorial du Windsor Yearbook of Access to Justice et le lecteur externe.

Département de sciences juridiques de l'Université du Québec à Montréal.

2 Nadja Alexander, Global Trends in Mediation, Alphen aan den Rijn (Pays-Bas), Kluwer Law International, 2006, à la p 2.

3 Nadja Alexander, supra note 2 à la p 1.

4 Owen Fiss, « Against Settlement », (1984) Yale L J 1073; Laura Nader, « Controlling Processes in the Practice of Law: Hierarchy and Pacification in the Movement to Re-Reform Dispute 
ils ne seront pas repris ici. Toutefois, malgré les critiques, la médiation extrajudiciaire est devenue une pratique très répandue au $\mathrm{XXI}^{\mathrm{e}}$ siècle, non seulement en Amérique du Nord mais aussi ailleurs sur la planète. Il est admis par la doctrine qu'elle peut, sous certaines conditions, constituer un moyen valable d'accès à la justice ${ }^{6}$. La Loi sur la médiation populaire de République populaire de Chine du 28 août 2010 est entrée en vigueur le $1^{\mathrm{er}}$ janvier $2011^{7}$. La médiation est un mode de résolution des conflits prévu par divers instruments dont la Loi sur la procédure civile de République populaire de Chine ${ }^{8}$. Toutefois, cette loi encadre la médiation judiciaire et non la médiation extrajudiciaire. Dans cette perspective, la Loi sur la médiation, qui s'applique essentiellement aux justiciables chinois du monde rural en raison des échelons d'administration territoriale visés, marque un développement important dans la mesure où il s'agit de la première loi encadrant la médiation extrajudiciaire en Chine, jusqu'alors régie par divers instruments juridiques dont des directives, opinions et interprétations de la Cour populaire suprême'. En outre, la Loi sur la médiation s'inscrit dans un contexte plus large, où les autorités judiciaires chinoises, étroitement contrôlées par le Parti communiste chinois (PCC), mettent l'accent sur la médiation, judiciaire et extrajudiciaire, comme mode de règlement des conflits. Les développements récents relatifs à la médiation judiciaire ont déjà fait l'objet de publications en langues occidentales mais pas ceux portant sur la Loi sur la médiation ${ }^{10}$. Or, ce renouveau de la médiation populaire en Chine soulève des questions. Tout d'abord, quels facteurs rendent compte de ce développement ? Plus précisément, s'agit-il pour le PCC de remédier à des problèmes, par ailleurs sérieux, d'accès à la justice chinoise en recourant, à l'instar d'autres États, aux nouveaux modes de règlement des conflits ? Quels liens, s'il y en a, peuvent être établis entre ce renouveau de la médiation et le mot d'ordre du régime résidant dans la construction d'une « société harmonieuse » fondée sur des «valeurs chinoises » et une tradition largement réinventée ${ }^{11}$ ? Enfin, ce nouveau régime de médiation

Ideology », (1993) 9 Ohio St J on Disp Resol 1 ; Marc Galanter, « Most Cases Settle : Judicial Promotion and Regulation of Settlements », (1994) 46 Stan L Rev 1339.

5 Nadja Alexander, supra note 2 à la p 4 ; Fan Yu et Li Hao, Jiufen Jiejue-Lilun, Zhidu yu Zhineng [La résolution des conflits : théories, système et capacité], Beijing, Presses de l'Université Qinghua, 2010, à la p 299.

6 Roderick A. Macdonald, « Access to Justice and Law Reform », (1990) 10 Windsor Y B Access Just 287, à la p 319 ; Yash Gai et Jill Cottrell, « The Rule of Law and Access to Justice », dans Yash Gai et Jill Cottrell, dir., Marginalized Communities and Access to Justice, New York, Routledge Cavendish, 2010, 1 à la p 3 ; Nadja Alexander, supra note 1 à la p 5.

7 Comité permanent de l'Assemblée populaire nationale, The People's mediation law of the People's Republic of China, 28 août 2010, en ligne : lawinfochina.com <http://www.law-infochina.com/display.a-s-p-x?id=8266-\&lib=law $>$ [Loi sur la médiation $]$.

8 Comité permanent de l'Assemblée populaire nationale, Civil Procedure Law of the People's Republic of China (9 avril 1991, modifiée le 28 octobre 2007), en ligne : lawinfochina.com $<$ http://www.law-info-china.com/display.aspx?id=6459\&lib=law\#>. Les articles visant la médiation sont au chapitre 8 , aux pp 85 à 91 .

9 L'expression « médiation populaire » sera employée dans le présent texte pour désigner la médiation extrajudiciaire.

10 Karl F. Minzner, « China's Turn Against the Law », en ligne: SSRN <http://ssrn.com/abstract $=1767455>$.

11 Sur ce thème de la tradition réinventée, voir le numéro de la revue Perspectives Chinoises qui lui est consacré : (2007) 4 Perspectives Chinoises. 
populaire constitue-t-il un apport pour l'accès à la justice des justiciables chinois ? Telles sont les questions traitées dans le présent texte.

\section{LE CONTEXTE DE LA MÉDIATION EXTRAJUDICIAIRE EN CHINE : LA RHÉTORIQUE DE LA SOCIÉTÉ HARMONIEUSE}

La République populaire de Chine a été fondée le $1^{\mathrm{er}}$ octobre 1949. En raison des contraintes d'espace, la période maoïste (1949-1976) ne sera pas abordée. Durant ces années, diverses raisons tenant au contexte international et interne de la Chine ont fait que cette dernière est demeurée largement fermée aux pays occidentaux. Après la mort de Mao Zedong en 1976 et un court intermède politique, Deng Xiaoping dirige le pays et lance en 1978 la politique de réformes et d'ouverture. La Chine s'ouvre à nouveau au monde, de manière sélective. Dans cette foulée, les réformes juridiques occupent une place importante. Initiées par le PCC, elles ont reposé sur divers mots d'ordre ou slogans. Jianfu Chen témoigne de l'importance de ces mots d'ordre du PCC car ils servent à façonner les réformes juridiques. En effet, les juristes chinois prennent appui sur les divers mots d'ordre du Parti pour justifier des réformes dans divers domaines du droit ${ }^{12}$. Du milieu de la décennie 1990 jusqu'au milieu de la décennie 2000, les réformes juridiques ont été guidées par le mot d'ordre de la « construction d'un État de droit socialiste ». Les débats autour de ce thème ont nourri une riche doctrine chinoise et occidentale depuis le milieu de la décennie $1990^{13}$. Ce mot d'ordre a rallié un nombre important de juristes chinois, qui, tout en étant conscients des limites de la transposition de l'État de droit ou de la Rule of Law dans le contexte chinois, ont vu dans cet idéal proclamé une base à partir de laquelle travailler ${ }^{14}$. Durant ces années, le PCC a lui-même encouragé les justiciables chinois à faire valoir leurs droits devant les tribunaux ${ }^{15}$. Or, ce mot d'ordre n'a plus cours. Le PCC a effectué un changement radical de paradigme qui se manifeste dans sa rhétorique sur la « construction de la société harmonieuse ». À celle-ci correspond un changement à l'égard du mode de résolution des conflits. La médiation, tant extrajudiciaire que judiciaire, fait l'objet d'une ardente promotion par le PCC et les autorités judiciaires.

12 Jianfu Chen, « The Transformation of Chinese Law: From Formal to Substantial », (2007) 37 Hong Kong L.J. 689, aux pp 720-725 [Chen, « Transformation »]. Jianfu Chen illustre cette dynamique en employant des expressions comme «économie socialiste de marché » et « établir un État de droit socialiste».

13 Voir, entre autres : en chinois les travaux de Li Buyun, Cai Dingjian et He Weifang ; en anglais, les travaux de Randall Peerenboom et Stanley B. Lubman ; en français, les travaux de Jean-Pierre Cabestan et Stéphanie Balme.

14 Xianchu Zhang, «Civil Justice Reform with Political Agendas », dans Yu Guanghua, dir., The Development of the Chinese Legal System. Change and Challenges, Londres (R-U), Routledge, 2011, 253, à la p 254.

15 Mary E. Gallagher, «Mobilizing the Law in China : «Informed Disenchantment » and the Development of Legal Consciousness » (2006) $40: 4$ Law and Society Review 783, à la p 794 ; Zheng Yongnian, « Du gouvernement par la loi à l'État de droit ? L'évolution de l'environnement juridique en Chine », (1999) 54 Perspectives Chinoises 31, à la p 40. 


\section{A. L'harmonie comme fondement de l'appel aux ressources endogènes et de la tradition réinventée}

En réalité, il s'agit d'une réactualisation de cette rhétorique car la référence à l'harmonie sociale trouve ses racines dans toutes les écoles de pensées en Chine mais surtout dans le confucianisme ${ }^{16}$. Ces développements se situent aussi dans le renouveau du confucianisme promu par le régime depuis le milieu de la décennie $1990^{17}$. L'harmonie sociétale est l'un des fondements du confucianisme et vise non seulement les relations entre les êtres humains mais entre ceux-ci et l'univers ${ }^{18}$. Peerenboom souligne qu'en Chine, historiquement, la politique a été conçue comme l'art d'atteindre l'harmonie sociétale ${ }^{19}$. C'est ici qu'intervient, dans le confucianisme, l'harmonie comme concept normatif. L'harmonie implique d'unifier les intérêts divers de la société en un tout cohérent. C'est le rôle des dirigeants chinois depuis l'ère impériale jusqu'au PCC aujourd'hui ${ }^{20}$. Or, le PCC fonde une partie de sa légitimité dans sa capacité à unifier harmonieusement les intérêts du peuple et de l'État ${ }^{21}$.

La rhétorique de l'harmonie opère doublement dans les discours et les pratiques chinois. Tout d'abord, dans la sphère politique où elle sert à justifier un ensemble de mesures mises sur pied par le PCC afin de maintenir la stabilité sociale. Des contraintes d'espace nous forcent à n'évoquer que brièvement ces mesures. La société harmonieuse marque un idéal qui « fait référence à un ordre socio-économique où tous les Chinois coexistent dans la paix et l'harmonie. Il implique l'élimination des conflits et contradictions au sein de la société qui se sont manifestés à travers les 87000 «incidents de masse »...de $2005 »^{22}$. Il ressort de l'énoncé de programme du PCC sur la société harmonieuse une très grande préoccupation par rapport à la nécessité de maintenir la stabilité sociale afin que la Chine puisse prospérer ${ }^{23}$. Le Parti a donc mis sur pied un ensemble de mesures politiques destinées à la mise en œuvre de la société harmonieuse ${ }^{24}$. Celles qui nous intéressent plus particulièrement ici ont trait à la mise sur pied, par les autorités centrales et régionales, de divers mécanismes de médiation ayant pour but de désamorcer les contradictions, ou conflits, de la société chinoise. Ces développements se situent aussi dans le renouveau du confucianisme promu par le

16 Randall Peerenboom, « Confucian Harmony and Freedom of Thought : the Right to Think Versus Right Thinking » dans Wm. Theodore de Bary et Tu Weiming, dir., Confucianism and Human Rights, New York, Columbia University Press, 1998, 234, à la p 238 [Peerenboom, « Confucian Harmony »].

17 Ibid. aux pp 236-237.

18 Guo-ming Chen, « The Impact of Harmony on Chinese Conflict Management », dans Guo-Ming Chen et Ringo Ma, dir., Chinese Conflict Management and Resolution, Westport, Londres, Ablex Publishing 2002, 3, à la p 5 [Chen, « The Impact of Harmony »].

19 Peerenboom, «Confucian Harmony », supra note 16 à la p 244.

20 Ibid.

21 Ibid à la p 240.

22 Willi Wo Lap-Lam, « Le dilemme du gouvernement Hu Jintao : la construction d'une société harmonieuse est-elle possible sans réformes politiques? », (2007) 3 Perspectives Chinoises 4, à la p 6. Les « incidents de masse » désignent en réalité les manifestations et les émeutes.

23 David Shambaugh, China's Communist Party. Atrophy and Adaptation, Washington, Berkeley; Woodrow Wilson Center Press, University of California Press, 2008, à la p 116.

24 Lap Lam, supra note 22 aux pp 6-8. Ces mesures visent entre autres l'efficacité administrative et la gestion des crises. 
régime $^{25}$. L'harmonie sociétale est l'un des fondements du confucianisme et vise non seulement les relations entre les êtres humains mais entre ceux-ci et l'univers ${ }^{26}$.

La rhétorique de l'harmonie se déploie également sur le terrain juridique où elle participe de la tradition réinventée. Il existe en Chine un débat qui oppose les tenants des valeurs universelles à ceux qui défendent des valeurs chinoises ${ }^{27}$ dans le contexte du renouveau nationaliste promu par le régime chinois ${ }^{28}$. Ce dernier se traduit, dans la sphère universitaire, par le mouvement pour les études nationales (gиохие). Ce mouvement a pris de l'ampleur depuis le milieu de la décennie 1990, même s'il est présent en Chine depuis le début du $\mathrm{XX}^{\mathrm{e}}$ siècle $^{29}$. Il revendique l'indigénisation des sciences humaines en Chine, ce qui implique d'employer des cadres conceptuels issus de la tradition chinoise plutôt que de l'Occident ${ }^{30}$. Les tenants de ce mouvement, qui se décline sous plusieurs modes, ont pour position commune ce qu'ils désignent comme l'hégémonie des cadres conceptuels occidentaux dans le monde des sciences humaines et ce, à l'échelle de la planète ${ }^{31}$. Cette prise de position repose sur la conviction que l'emploi des cadres conceptuels occidentaux ne rend pas justice aux sciences humaines chinoises et en déforme la véritable compréhension, car ils ont été élaborés dans un tout autre contexte. En fait, nous sommes devant une version du guoxue qui voit celui-ci comme «fondement de valeurs indigènes à l'ère de la mondialisation » ${ }^{32}$. Ces raisonnements ont été repris par certains juristes chinois qui les ont transposés dans la sphère des recherches sur le droit chinois. Ainsi, Zhu Suli a lancé en 1996 son appel au bentuhua, ou appel aux paradigmes locaux, afin de fonder les réformes juridiques entamées depuis $1978^{33}$. Le mouvement en faveur des paradigmes locaux se décline sous plusieurs modes ${ }^{34}$. La version dominante, véhiculée par Zhu Suli, revêt des dimensions nationalistes très en phase avec les propos du régime chinois. Les réformes juridiques chinoises font appel aux transferts de droit. La faculté de pouvoir puiser dans les modèles juridiques occidentaux a été, au début des réformes, vécue comme une véritable libération intellectuelle par les juristes chinois emprisonnés jusqu'alors dans la seule catégorie du «droit soviétique » ${ }^{35}$. Toutefois, la place des modèles juridiques occidentaux comme source d'inspiration du droit chinois légiféré a commencé à

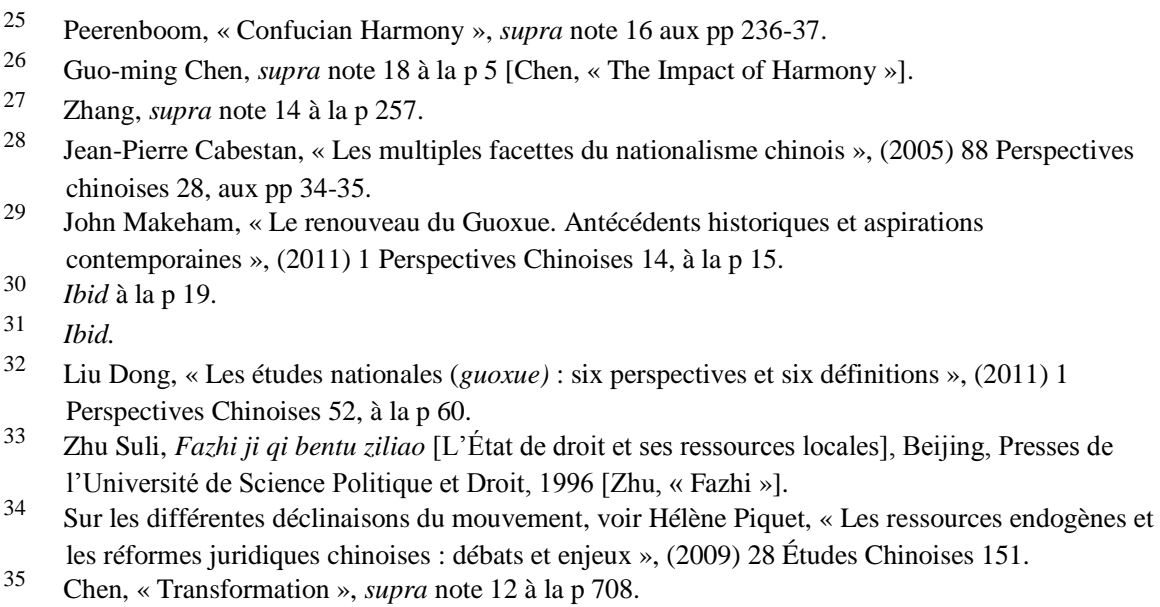


susciter des réactions, demeurées minoritaires, au milieu de la décennie 1990. Initialement limité à la sphère universitaire, voilà que l'appel aux paradigmes locaux ou ressources endogènes trouve une résonnance chez les autorités judiciaires chinoises elles-mêmes ${ }^{36}$. Or, ici intervient le processus de la réinvention de la tradition fondé sur la rhétorique de l'harmonie. En effet, la référence à l'harmonie alimente un discours des autorités judiciaires et d'une partie importante de la doctrine chinoise. Les ressources endogènes de la tradition juridique chinoise sont recréées ou, à tout le moins, substantiellement réinterprétées afin de fonder un nouvel ordre juridique.

D'une part, la référence à l'harmonie est employée afin de créer une représentation des justiciables chinois comme ayant toujours répugné à la perspective d'un procès au nom d'une supposée préférence culturelle pour $l^{\text {'harmonie }}{ }^{37}$. Le procès revêt ainsi la connotation négative de mode conflictuel de résolution des conflits. Les justiciables chinois préfèreraient céder (rang) plutôt que de faire valoir leurs droits et ainsi rompre l'harmonie sociétale plus précieuse que tout. Cette représentation a cours, autant pour la Chine impériale que pour la Chine des réformes (depuis 1978) ${ }^{38}$. Or, elle a été démentie par des chercheurs faisant autorité. Commençons par la Chine impériale. Cette vision essentialiste des justiciables chinois a été démontée par les travaux de David Buxbaum, puis plus tard de Philip Huang, sur la Chine impériale ${ }^{39}$. En résumé, les justiciables chinois recouraient certes à la médiation extrajudiciaire pour résoudre certains de leurs conflits $^{40}$. Toutefois, ils usaient abondamment du système judiciaire étatique, très développé, et ce, autant en ville qu'à la campagne ${ }^{41}$. S'il est vrai que le confucianisme véhicule une certaine aversion pour les procès, il existe, selon les termes mêmes de Huang, une rupture entre les représentations et les pratiques des justiciables chinois à l'ère impériale ${ }^{42}$. Plusieurs historiens chinois du droit chinois ont également mis cela en lumière.

En ce qui concerne la période des réformes, depuis 1978, certaines précisions sont de mise. L'apparition de la représentation du justiciable chinois comme porté à faire prévaloir la sauvegarde de l'harmonie sur ses droits est d'origine récente dans la doctrine chinoise. Elle remonte au début de la décennie 2000. Le débat porte surtout sur les justiciables chinois du monde rural. Les auteurs s'accordent pour affirmer que le système judiciaire étatique a été sous-utilisé dans le monde rural durant la décennie 1990. Les divergences de vues résident dans les

\footnotetext{
Zhang, supra note 14 à la p 254.
}

Goh Bee Chen, Law Without Lawyers, Justice Without Courts. On Traditional Chinese Mediation, Aldershot (RU), Royaume-Uni, 2002, aux pp 8-9 [Goh, « Law Without Lawyers »]. Ibid à la p 26.

39 David Buxbaum, « Some Aspects of Civil Procedure and Practice at the Trial Level in Tanshui and Hsinchu from 1789 to 1895 », (1971) 30:2, The Journal of Asian Studies 255 ; Philip C.C. Huang, Civil Justice in China. Representation and Practice in the Qing, Stanford, Stanford University Press, 1996.

40 Huang, supra note 39 aux pp 61-68.

41 Buxbaum, supra note 39 aux pp 270-271. Tout son article présente des cas soumis aux tribunaux judiciaires pendant la période étudiée. Philip C. C. Huang, Chinese Civil Justice, Past and Present, London, New York, Rowman \& Littlefield Publishers, 2010. Voir plus particulièrement aux pp 152-154, où il démonte les représentations confucéennes du rapport au droit des justiciables chinois. 
explications qui sont avancées. Zhu Suli ${ }^{43}$ met de l'avant des causes d'ordre culturel, alors que Fu Hualing ${ }^{44}$ et Ying Xing ${ }^{45}$ mettent de l'avant des causes surtout, mais non exclusivement, économiques dans une perspective d'accès à la justice. Pour Zhu Suli, les décisions rendues par les tribunaux étatiques à l'issue de procès tenus dans le monde rural seraient incomprises car en rupture avec le sens de la justice des justiciables, qui n'est pas pris en compte dans le processus décisionnel des juges ${ }^{46}$. En outre, ces justiciables du monde rural auraient une faible conscience du droit et en tous les cas, ne comprendraient pas le droit édicté par l'État. Song Wuchao abonde dans le même sens et ajoute que les justiciables du monde rural chérissent l'harmonie avant tout, ce qui les prédispose à régler leurs conflits par la médiation extrajudiciaire ${ }^{47}$. Fu Hualing avance d'autres explications. Il rappelle d'abord que les tribunaux populaires de base instaurés dans le monde rural dans la décennie 1980 ont connu un taux élevé d'utilisation ${ }^{48}$. Selon les statistiques officielles, entre 1988 et 1991, 80 p. 100 des causes étaient traitées par les tribunaux judiciaires ${ }^{49}$. Par la suite, leur taux d'utilisation a baissé substantiellement. Selon Fu, cette baisse s'explique par la décentralisation et la diminution des ressources qui en ont découlé dans les échelons territoriaux du monde rural. Les districts et villages connaissent une situation de crise financière. En réalité, ce sont ces autorités locales qui doivent financer l'appareil judiciaire et elles ne le font que très peu. Il en résulte que le système judiciaire va chercher le manque à gagner directement auprès des justiciables du monde rural (transfert de la crise financière et des coûts vers ceux-ci), sous forme de frais dont certains sont légaux (frais de justice) et d'autres illégaux (les cadeaux). Ils s'ajoutent à la perte de revenus pour le temps non travaillé et aux frais de transport L'ensemble des frais à débourser demeure hors de la portée du paysan ordinaire ${ }^{50}$. Cela étant, Fu affirme que la conscience d'être titulaires de droits à défendre habite les justiciables du monde rural ${ }^{51}$. Entre autres, le contact des mingong (travailleurs migrants issus du monde rural) avec la ville renforce encore leur conscience du droit ${ }^{52}$. Fu affirme que s'ils ne saisissent pas toujours le système judiciaire étatique pour faire valoir leurs droits, les paysans sont cependant très conscients des violations de ces droits et réagissent par une panoplie de moyens qui varient

43 Zhu Suli, « Guanyu nengdong sifa yu da tiaojie », (2010) $1: 153$, Zhongguo Faxue 5 [Zhu, « Tiaojie»].

44 Fu Hualing, « Cong xiangtu shehui falü zhidu kan falü yu fazhan: chongfen de jiejue yu jingji fazhan » [Regards sur le droit et le développement à partir de la société rurale], dans Liang Zhiping, dir., Guojia, shichang, shehui: dangdai zhongguode falü yu fazhan [L’État, le marché, la société : le développement du droit de la Chine contemporaine], Beijing, Presses de l'Université de Science Politique et Droit, 2005, 141 [Fu, « Xiangtu Shehui »].

45 Ying Xing, "“Yingfa ru Xiang”, "Jiejin Zhengyi”- Dui Zhongguo XiangCun Chijiao Lushi de ge an Yanjiu » [« Dissemination of Law at Village Level » and « Access to Justice » - A Case Study of « Barefoot Lawyers » in China ], (2007) 25:1, Zhengfa Luntan 79.

46 Zhu, « Tiaojie », supra note 43 à la p 7.

47 Song Wuchao, Tiaojie lifa yanjiu [Recherches sur la médiation], Beijing, Presses de l'Université de Science Politique et Droit, 2008, à la p 17.

$48 \mathrm{Fu}$, « Xiangtu Shehui », supra note 44 à la p 149.

49 Ibid.

$50 \quad$ Ibid à la p 156.

51 Ibid à la p 160.

52 Ibid à la p 161. 
selon la nature et l'intensité du conflit : pression exercée sur les autorités locales, suicide, pétitions ${ }^{53}$. Par ailleurs ils ont pris l'habitude d'invoquer le droit étatique dans la formulation de leurs revendications, ce qui donne un caractère légal et légitime à leurs demandes ${ }^{54}$.

Les travaux de Ying Xing apportent aussi un éclairage complémentaire ${ }^{55}$. Ying Xing a directement pris le contrepied de Zhu Suli en démontrant que les justiciables chinois du monde rural n'aspirent pas à vivre hors du système judiciaire étatique mais bien à en user, pour peu qu'on leur en donne les moyens. Dans son étude sur les avocats aux pieds nus dans le monde rural, Ying Xing fait plutôt état de la quête de droit étatique par les justiciables du monde rural chinois. Ces avocats aux pieds nus représentent les justiciables ruraux devant les tribunaux dans certains types de cause, assez simples, surtout en droit administratif. Ils connaissent un succès important dans le monde rural précisément parce qu'ils servent de lien entre les justiciables du monde rural et le système judiciaire étatique. Ils apportent ainsi un certain remède à la pénurie de ressources juridiques qui sévit en monde rural, dont les avocats en titre ${ }^{56}$.

D'autre part, en corollaire de cette représentation du justiciable chinois adoptant toujours une attitude non contentieuse face à un conflit, l'harmonie sert aussi à justifier le renouveau de la médiation, tant judiciaire qu'extrajudiciaire ${ }^{57}$. En effet, en raison de sa nature non antagoniste, la médiation devient le mode tout désigné de résolution des conflits pour des justiciables soucieux de préserver des relations harmonieuses une fois leur conflit résolu ${ }^{58}$. Elle est érigée au rang de ressource endogène. Ce discours devenu dominant consiste en une réaction contre le modèle «importé » (lire : occidental) de la justice procédurale et du procès Ainsi, depuis le début des réformes juridiques en cours, une trop grande place serait consentie aux modèles juridiques étrangers au détriment des ressources endogènes, qu'il convient de réhabiliter ${ }^{60}$. En outre, ces règles et institutions importées ne seraient pas compatibles avec les besoins des justiciables chinois, ces

53

Ibid

auteurs ont exploré plus à fond ce phénomène qu'ils désignent par l'expression « rightful resistance » ou résistance légitime. Voir Kevin J. O’Brien et Lianjiang Li, Rightful Resistance in Rural China, New York, Cambridge University Press, 2006. Tout leur ouvrage est consacré aux diverses demandes des paysans chinois qui protestent contre tel ou tel abus.

58

59

chinois témoignent de l'existence d'une riche tradition juridique en Chine impériale (-221-1911)

et on trouve en droit chinois impérial un ensemble très élaboré de règles qui régissent la détermination de la peine et le procès, notamment en matière pénale. Jérôme Bourgon, « Principe de légalité et règle de droit dans la tradition juridique chinoise », dans Mireille Delmas-Marty et Pierre-Étienne Will, dir., La Chine et la démocratie, Paris, Fayard, 2007, 157. Voir notamment la discussion, à la p 169, sur les règles édictées afin que le jugement soit conforme aux faits et à la loi. besoins des gens ordinaires], (2006) 5 Faxuejia 130, à la p 136. 
derniers préférant des modes non contentieux de règlement des conflits ${ }^{61}$. De surcroît, les justiciables chinois ne comprendraient pas les concepts contenus dans le droit issu des transferts ${ }^{62}$. Plus précisément, les justiciables chinois ne saisiraient pas la différence entre la justice procédurale et la justice substantielle ${ }^{63}$. Ce droit étatique ne répondrait pas à leurs besoins et accuserait un déficit de légitimité ${ }^{64}$. Il faut donc fouiller la tradition chinoise afin de dégager les paradigmes locaux pouvant fonder les réformes juridiques. La justice procédurale et le procès, ces deux modèles dits étrangers à la tradition chinoise ${ }^{65}$, seraient difficilement reçus, particulièrement dans le monde rural, en raison de leur incompatibilité - tenant à leur origine «occidentale» (et urbaine) -, avec la tradition juridique chinoise censément caractérisée par une préférence culturelle pour la médiation comme mode de résolution des différends ${ }^{66}$. Cette dernière convient aux justiciables chinois et devrait donc être privilégiée comme mode non contentieux de résolution des conflits ${ }^{67}$. Zhu Suli n'hésite pas à affirmer sa conviction que la médiation populaire, comme mode de résolution des conflits, a une valeur infiniment supérieure au procès, en particulier pour les justiciables du monde rural ${ }^{68}$. Le juge You Tiancheng est aussi en faveur de la médiation populaire ${ }^{69}$. Ce mode de résolution des conflits permet selon lui de ménager les relations entre les parties en évitant la logique perdant-gagnant qui caractérise le procès devant le tribunal ${ }^{70}$. En effet, pour lui, le droit étatique comme instrument de règlement des conflits atteint des limites. Par rapport au procès, la médiation est présentée comme flexible dans son processus et efficace dans l'œuvre de pacification des parties. Cependant, You Tiancheng prend bien soin de poser des balises à la médiation extrajudiciaire. Elle ne peut se dérouler que si les parties y consentent librement ${ }^{71}$. Bien que les données dont nous disposons suggèrent que les autorités judiciaires chinoises voient aussi dans la médiation un moyen de désengorger les tribunaux ${ }^{72}$, la

61 Wu Zengji, « Guomin Xinli dui Zhongguo Fazhi Xiandaihua Daolu de Yinxiang ji qi Xiandai Jiazhi » [L'influence de la mentalité nationale sur la modernisation et les valeurs contemporaines du système judiciaire chinois], (2007) 2 Zhengfa Luntan 31, à la p 34.

62 Wang et Zhao, supra note 60 à la p 133.

63 Ibid à la $\mathrm{p} 134$.

64 Ibid à la p 132

65 Fan Yu et Li Hao, Jiufen Jiejue- Lilun, Zhidu yu Zhineng [La résolution des conflits : théories, système et capacité], Beijing, Presses de l'Université Qinghua, 2010, à la p III [Fan et Li, « Jiufen »].

66 Henan Sheng Faxuehui [Conférence de Théorie du droit de la province du Henan], Tiaojie Zhidu Lilun yu Shijian [Théorie et pratique du système de médiation], Zhengzhou, Presses de l’Université de Zhengzhou, 2010, à la p 45 ; Song Chao Wu, supra note 47, à la p 17.

67 Wu, supra note 61 à la p 36.

68 Zhu, « Tiaojie », supra note 43 à la p 7.

69 You Tiancheng, «Renmin Tiaojie yao de ming, jue de kai, zou de yuan » [La médiation populaire : absolument...], en ligne : Dongfangfayan

<http://www.dffy.com/faxueti/ss/201012/20101212114847.htm>. Avant de devenir juge, You Tiancheng était professeur de droit. Ses travaux portent sur le pluralisme juridique et le monde rural. Il est très connu en Chine, du fait de ses travaux.

70 Ibid. Les lignes qui suivent sont notre traduction des propos du juge You Tiancheng.

71 Ibid.

72 Fan Yu, Jiufen Jiejue de Lilun yu Shixian [Théorie et pratique de la résolution des conflits], Beijing, Presses de l’Université Qinghua, 2007, à la p 377. Fan Yu retrace la progression de ce 
doctrine reprend inlassablement le raisonnement du paradigme local. Outre le caractère non antagoniste de son processus, des auteurs soulignent aussi sa nature flexible et la plus grande facilité d'exécution des accords qui en sont issus, par rapport à une décision rendue par un tribunal judiciaire à l'issue d'un procès ${ }^{73}$. Une autre caractéristique de la médiation extrajudiciaire qui séduit certains auteurs chinois réside dans le fait qu'elle est fondée sur la protection des intérêts des parties plutôt sur leurs droits définis de manière étroite par la procédure ${ }^{74}$.

Tant pour Zhu Suli que pour You Tiancheng, la médiation populaire constitue indéniablement une ressource endogène utile aux réformes juridiques en cours. Ces positions des auteurs ont trouvé écho auprès des autorités judiciaires. Au milieu de la décennie 2000, la Cour populaire suprême a embrassé le concept de "justice harmonieuse » ${ }^{75}$. Ainsi, elle a édicté des Opinions et Avis, instruments juridiques propres à la Chine et revêtant une valeur contraignante, dans lesquels elle vante les mérites de la médiation ${ }^{76}$. Dans le contexte de la Chine du XXI ${ }^{\mathrm{e}}$ siècle, la médiation est promue au rang du paradigme local par excellence, tant dans son volet extrajudiciaire que judiciaire. Alors, la médiation, ressource endogène recréée, répond au sentiment nationaliste et gagne ainsi une légitimité difficilement contestable puisqu'elle est d'abord politique.

\section{B. La médiation populaire avant la réforme de 2011}

Quelques mots sur le système judiciaire étatique s'imposent avant d'aborder la médiation ${ }^{77}$. Au début de la politique de réforme et d'ouverture, la nouvelle équipe de dirigeants veut attirer les investissements étrangers et doit à cette fin réformer le droit légiféré. Mais outre cette motivation, les dirigeants d'alors veulent aussi amorcer une nouvelle ère, qui serait caractérisée par celle d'un gouvernement régi par la $10^{78}$. Il s'agit ici de marquer une rupture par rapport à la période maoïste, associée au règne de l'arbitraire par excellence et du "gouvernement par 1 'homme ${ }^{79}$. Dans cette perspective, le PCC amorce le processus de reconstruction

concept dans les divers instruments promulgués par la Cour populaire suprême [Fan, « Chongfen Jiejue »], aux pp 379-380.

73 Song, supra note 47 à la p 105.

74 Ibid à la $\mathrm{p} 49$.

75 Fan, « Jiufen Jiejue », supra note 72 à la p 377. Fan Yu retrace la progression de ce concept dans les divers instruments promulgués par la Cour populaire suprême.

76 Cour populaire suprême, Several Opinions of the Supreme People's Court on Further Displaying the Positive Roles of Litigation Mediation in the Building of a Socialist Harmonious Society (6 mars 2007); en ligne : <http://www.lawinfochina.com/display.aspx?id=5930\&lib=law> ; Cour populaire suprême, Notice of the Supreme People's Court on Issuing Several Opinions on Further Implementing the Work Principle of "Giving Priority to Mediation and Combining Mediation with Judgment (6 juillet 2010) ; en ligne : <http://www.lawinfochina.com/display.aspx?id=8397\&lib=law>

77 Comme cette étude porte sur la médiation extrajudiciaire, nous renvoyons les lecteurs à d'autres sources en ce qui a trait au système judiciaire étatique. Parmi celles-ci : Yu Guanghua, dir., The Development of the Chinese Legal System. Change and Challenges, Londres (R-U), Routledge, 2011, supra note 14 ; Margaret Y. K. Woo et Mary E. Gallagher, dir., Chinese Justice. Civil Dispute Resolution in Contemporary China, Cambridge (É.-U.), Cambridge University Press, 2011, et l'ouvrage codirigé par $\uparrow$ Cai Dingjian et Wang Chenguang (voir note suivante).

78 Wang Chenguang, « From the Rule of Man to the Rule of Law », dans † Cai Dingjian et Wang Chenguang (dir.), China's Journey Toward Rule of Law, Leide, Boston, Brill 2010 1, à la p 5.

79 Ibid. L'homme en question n'est autre que Mao Zedong. L'expression elle-même fait référence au débat qui a opposé les confucianistes, partisans du gouvernement par l'homme [de bien] et les 
du système judiciaire, rouvre les universités fermées pendant la Révolution culturelle (1966-1976) et ordonne aux juristes chinois de recourir aux transferts de droit pour élaborer le nouveau droit ${ }^{80}$.

Trait marquant des réformes, le recours aux tribunaux par les justiciables chinois n'a cessé de croître, surtout depuis la décennie $1990^{81}$. Les justiciables chinois, surtout dans le monde urbain, ont bien répondu à l'invitation d'user des tribunaux judiciaires pour défendre leurs droits. De plus, des données récentes confirment que les recours aux tribunaux, tous domaines confondus, sont à la hausse. Ainsi, le nombre de causes a augmenté de 25 p. 100 entre 2005 et 2009, créant chez les juges chinois le sentiment d'être la proie d'un déferlement incessant de dossiers à traiter ${ }^{82}$. En matière civile, l'augmentation est spectaculaire car leur nombre est passé de 300787 en 1978 à 5412591 en $2008^{83}$. C'est dire qu'en dépit de certains maux, le système judiciaire étatique revêt une légitimité certaine, aux yeux des justiciables chinois, comme forums de règlements des conflits $^{84}$. Durant toute cette période, si les autorités investissent beaucoup de ressources dans le système judiciaire, la médiation extrajudiciaire est pour sa part négligée. Cependant, même si les justiciables chinois se sont de plus en plus tournés vers les tribunaux étatiques comme forums de résolution des conflits, la médiation extrajudiciaire a coexisté, bien que dans l'ombre, avec ceux-ci. Sa pratique n'a jamais disparu et a fait l'objet de diverses tentatives de revitalisation de la part des autorités depuis le début des réformes.

Des limites d'espace nous contraignent à ne pas traiter de la médiation extrajudiciaire dans la Chine maoïste. L'étude la plus approfondie sur le sujet demeure celle faite par Stanley Lubman ${ }^{85}$. Si le modèle alors mis en place en 1954 reste sensiblement le même jusqu'à nos jours, il connaît, au fil du temps, des changements notables quant à ses objectifs et à son utilisation par les justiciables chinois. Une de ses caractéristiques est que la médiation extrajudiciaire fait partie intégrante d'un processus étatique, à multiple visages de règlement des conflits : les recours judiciaires, le système de pétitions et de médiation populaire sont tous des moyens développés par les dirigeants. Autre trait distinctif du régime public de médiation extrajudiciaire, ce dernier opère sur la supervision et avec l'aide des tribunaux populaires, surtout ceux dits « de base ». La médiation est effectuée par des comités de médiation populaire, composés de personnes agissant à titre de médiateurs. En théorie, de tels comités sont censés être créés à tous les échelons

légistes qui prônaient le gouvernement par la loi. Voir aussi : Fu Hualing et Roy Cullen, 2008, « From Mediatory to Adjudicatory Justice: the Limits of Civil Justice Reform in China »; en ligne : (2008) SSRN <http://s-s-r- n.com/ abstract=1306800>, aux pp 66-67.

80 Chen, « Transformation », supra note 12 aux pp 706-707.

81 Song, supra note 47 aux pp 44-45. Wei Luo, The Civil Procedure Law and Court Rules of the People's Republic of China, Buffalo, William \& Hein Co. Inc, 2066, aux pp 14-15.

82 China Daily, « Courts hit by a rising number of lawsuits », China Daily [Beijing] (14 juillet 2010); en ligne : Chinadaily.com.cn <http://www.chinadaily.com.cn/usa/201007/14/content_11020426.htm>. En 2005, les tribunaux ont instruit 8,37 millions de causes et, en 2009, 10,54 millions. Le nombre de juges reste stable.

83 China by Numbers 2010, Hong Kong, China Economic Publishing, 2010, à la p 90.

84 Song, supra note 47 aux pp 44-45. Song attribue cela à la diffusion du concept d'État de droit socialiste dans la société chinoise, qui valorise les recours aux tribunaux judiciaires.

85 Stanley B. Lubman, Bird in A Cage. Legal Reform in China After Mao, Stanford, Stanford University Press, 1999. Voir le chapitre 3 consacré à ce sujet, soit aux pp 40-70. 
territoriaux de la Chine, tant dans le monde rural que dans le monde urbain ${ }^{86}$. Toutefois, leur nombre varie selon les provinces.

Lubman signale qu'en 1989, le ministère de la Justice a décidé de redonner une nouvelle vie au réseau des médiateurs du monde rural. Ainsi, un nouveau règlement régissant la médiation populaire est promulgué en $1989^{87}$. Cette initiative reposait sur deux objectifs : le maintien de l'ordre public et l'allègement du fardeau des cours. Des comités de médiation populaire ont été revitalisés. En outre, des assistants judiciaires et des bureaux cantonaux de services juridiques ont vu le jour ${ }^{88}$. Ces développements institutionnels ont été de pair avec la politique du ministère de la Justice qui privilégiait d'abord la médiation comme mode de résolution des conflits ${ }^{89}$.

Avant la réforme de la Loi de République populaire de Chine sur la procédure civile $^{90}$, le recours à la médiation était obligatoire. La réforme de 1991 apporte un changement important, car il est alors stipulé à l'article 85 que la médiation doit être un processus volontaire, c'est-à-dire auquel les parties ont librement consenti ${ }^{91}$. Autres changements appréciables : le processus de médiation devient nettement moins politisé que durant la période maoïste et les médiateurs peuvent faire plus de place à la règle de droit dans leur répertoire normatif ${ }^{92}$. Cependant, en pratique, selon l'étude de Liu Guangan et de Li Cunpeng, la règle de droit a été moins invoquée par les médiateurs que les sentiments et la raison ${ }^{93}$. Ils ont effectué une enquête de terrain dans plusieurs provinces, incluant des provinces habitées par des minorités. Ils ont constaté que les médiateurs ont fait une large place aux qingli, soit les sentiments et le sens local de ce qui est raisonnable ${ }^{94}$. Les cas faisant appel aux qingli sont nettement plus nombreux que les cas reposant sur des normes juridiques. Par ailleurs, dans les provinces habitées par des minorités, les médiateurs ont parfois fait place à la coutume locale afin de résoudre le conflit ${ }^{95}$. Certains cas ont aussi fait appel, en même temps, aux qingli et au droit de manière complémentaire $^{96}$. En réalité, les médiateurs ont souvent combiné plusieurs normes revêtant divers degrés de valeur persuasive.

86 Plusieurs instruments juridiques précédant la Loi sur la médiation populaire contiennent des dispositions en ce sens.

87 Conseil des Affaires d'État, Renmin Tiaojie Weiyuanhui Zuzhi Tiaoli [Règlement sur les comités populaires de médiation], 1989, dans Wu Jun Ying, dir., Renmin Tiaojie Fagui Huibian yu Dianping [Compilation des règlements sur la médiation populaire], Beijing, Presses Juridiques de Chine, 2011, 29.

Liu Guang'an et Li Cunpeng, « Minjian tiaojie yu quanli baohu » [La médiation populaire et la protection des droits], dans Xia Yong, dir., Zou xiang quanli de shidai [En marche vers l'âge des droits], $2^{\mathrm{e}}$ éd., Beijing, Presses de l'Université de Science Politique et Droit, 1999, 251-90, aux pp 259-260

Ibid aux pp 266-268. 
Sur le plan institutionnel, les comités de médiation populaire sont des organisations de masse situées administrativement sous l'échelon du village et des comités de quartiers mais opérant sous la supervision des gouvernements populaires et des cours populaires ${ }^{97}$. Dans le monde rural, les membres du comité sont élus par le public ${ }^{98}$. Il s'agit d'habitants du village, à la bonne réputation, possédant une certaine influence dans la communauté de même qu'une intégrité morale $^{99}$. Selon Fan Yu, la médiation est d'abord une affaire d'expérience et ne requiert pas de formation en droit pour les médiateurs, à l'exception du directeur du comité de médiation ${ }^{100}$.

Le processus de médiation est gratuit et la rémunération des médiateurs est censée être versée par le comité de villageois, ou de quartier ${ }^{101}$. Le directeur du comité de médiation travaille à temps partiel et reçoit une compensation du village pour son travail $^{102}$. Chaque village est divisé en unités de 10 foyers pour lesquels il existe un médiateur. Ce dernier doit traiter par médiation les conflits jugés mineurs et faire rapport aux directeurs du comité de villageois ${ }^{103}$. Dans son étude de terrain, Fu Hualing a constaté la nature théorique du caractère gratuit de la médiation, car, en réalité, les médiateurs facturent des frais pour leur travail. Ils se sentent d'autant plus autorisés à le faire que le gouvernement ne finance pas le régime ${ }^{104}$. Déjà, avant la réforme de 2011 , la rémunération adéquate des médiateurs fait problème. Fan Yu estime que les comités de médiation remplissaient des fonctions utiles dans la décennie 1990 et au début du XXI ${ }^{\mathrm{e}}$ siècle. Dans cette perspective, elle déplore le manque de financement du régime ${ }^{105}$.

Fu Hualing signale que la fonction première des comités de médiation et des assistants judiciaires (voir ci-dessous) consiste à supprimer les conflits et à empêcher que ceux-ci ne débordent les limites de la localité ${ }^{106}$. Fan Yu signale que parfois, pour tenter de résoudre un conflit, les comités de médiation doivent unir leurs efforts à ceux d'autres institutions locales comme les clans ou les associations religieuses ${ }^{107}$. Lorsque le processus de médiation aboutit à un accord, ce dernier n'est habituellement pas formalisé par un écrit ${ }^{108}$.

En Chine, il n'existe pas de véritable séparation des pouvoirs, ce qui implique que les pouvoirs du PCC et du gouvernement ne sont pas délimités les uns par rapport aux autres. Cependant, ils ne possèdent pas la même étendue de pouvoir.

97 Fu Yulin, « Dispute Resolution and China’s Grassroots Legal Services », dans Margaret Y. K. Woo et Mary E. Gallagher, dir., Chinese Justice. Civil Dispute Resolution in Contemporary

98 China, Cambridge (ÉU), Cambridge University Press, 2011, 314, à la p 321.

99 Fan Yu, «Jiufen jiejue... », supra note 72 à la p 521.

$100 \quad$ Ibid à la p 533.

101 Fu Yulin, supra note 97 à la p 321.

102 Fu Hualing, «Shifting Landscape of Dispute Resolution in Rural China », dans Jianfu Chen, Yuwen Li et Jan Michiel Otto, dir., Implementation of Law in the People's Republic of China, The Hague, Kluwer Law International, 2002, 178, à la p 179 [Fu, « Shifting Landscape »].

103 Ibid à la $\mathrm{p} 182$.

104 Fu Hualing, « Shifting Landscape », supra note 102 à la p 187.

105 Fan Yu, «Jiufen jiejue », supra note 72 aux pp 520-521.

106 Fu Hualing, «Shifting Landscape », supra note 102 à la p 189.

107 Fan Yu, «Jiufen jiejue », supra note 72 à la p 532.

108 Ibid à la p 533. 
Sur le terrain, le comité du parti aura préséance sur le gouvernement et sur l'assemblée populaire ${ }^{109}$. Dans le contexte du monde rural, la justice rendue par les comités de médiation demeure largement influencée par le PCC et le gouvernement local ${ }^{110}$. À titre d'exemple, le directeur du comité de médiation est souvent le directeur du comité de villageois (instance de gouvernance des villages) ou encore, le directeur du bureau local de la sécurité publique ${ }^{111}$.

Les travaux de Neil Diamant, couvrant la période maoïste et celle des réformes (depuis 1978) ${ }^{112}$, apportent un éclairage sur les réalités de la pratique de la médiation. En plus d'entrevues avec divers acteurs de la médiation à l'époque, dont des justiciables, il a eu accès à des archives. Il a ainsi étudié des cas de médiation dans les mondes rural et urbain. Au cours de son enquête, Diamant a recueilli plusieurs informations sur les personnes agissant à titre de médiateurs et les perceptions qu'en ont eu et en ont encore les justiciables chinois. Ici interviennent des facteurs politiques. Le PCC a enclenché une véritable révolution sociale qui s'est traduite, entre autres, dans le monde rural, par l'élimination et le départ des anciennes élites. Les personnes ayant été promues cadres du Parti étaient souvent très peu éduquées, détenant, au mieux, un diplôme d'études secondaires. Or, ces mêmes cadres siégeaient souvent aux comités de médiation et étaient appelés à agir comme médiateurs. Ces villageois étaient jeunes (entre 20 et 25 ans), de sexe masculin, connus pour leurs comportements machistes ${ }^{113}$. Ces jeunes cadres ne possédaient aucune expérience à titre de médiateurs et, souvent, les résultats de leurs séances de médiation déplaisaient aux deux participants. Diamant a en outre constaté que les médiateurs avaient tendance à renvoyer directement les acteurs du conflit devant les tribunaux. À la lumière de ces faits, Diamant affirme que les comités villageois de médiation étaient souvent peu fonctionnels ${ }^{114}$. En outre, un autre facteur a contribué à détourner les justiciables ruraux des nouveaux médiateurs. Ces derniers possédaient une trop grande connaissance des acteurs du conflit et cette connaissance, loin de nourrir la confiance des justiciables, suscitait au contraire une crainte de partialité de la part des médiateurs. Un schéma assez constant ressort des enquêtes de Diamant, soit que les femmes ont le plus souvent catégoriquement refusé de faire affaire avec les médiateurs, par crainte de leurs préjugés machistes et autres, pour aller saisir directement les tribunaux. Ici, c'est toute la logique de proximité du monde rural qui est en cause. Loin de rapprocher les justiciables, elle les divise.

La renaissance des comités de médiation populaire du monde rural a été accompagnée de l'élimination, en 1981, du système des assistants judiciaires chargés de les superviser (fonction qui revenait jusqu'alors aux tribunaux). Les assistants judiciaires sont définis comme des personnes chargées de l'administration de la justice au sein du gouvernement populaire, soit du canton. Toutefois, ils ne sont pas considérés comme une institution de l'État et leur statut

109 Jean-Pierre Cabestan, «Les systèmes politiques du monde chinois », dans Guy Faure, Nouvelle géopolitique de l'Asie, Paris, Ellipses, 2005, 183, à la p 193.

110 Lubman, supra note 85 à la p 229.

111 Fu Yulin, supra note 97 à la p 337.

112 Neil J. Diamant, «Conflict and Conflict Resolution in China: Beyond Mediation-Centered Approaches », (2000) $44:$ 4, The Journal of Conflict Resolution, 523, à la p 524.

113 Ibid à la p 532.

114 Ibid à la p 533. 
réel demeure précaire ${ }^{115}$. Ils participent directement à la médiation tout en en supervisant le processus, servant de lien entre les gouvernements populaires et les comités de médiation $^{116}$. En d'autres termes, le travail des comités de médiation ne se fait pas sur une base d'indépendance par rapport aux autorités locales ${ }^{117}$, qu'il s'agisse du PCC ou du gouvernement. Fu Hualing estime que l'institution des assistants judiciaires n'a pas été prise au sérieux par les gouvernements locaux.

Une autre institution a vu le jour dans le monde rural en 1987. Il s'agit des bureaux de services juridiques des cantons et villages (BSJCV). Ces derniers opèrent sous la direction des gouvernements locaux et des assistants judiciaires. Ils peuvent agir comme médiateurs ou représentants des parties dans le processus de médiation ou les actions en justice ${ }^{118}$. Ils sont appelés à fonctionner comme une entreprise, devant produire leurs propres revenus ${ }^{119}$.

Dans le monde urbain, les comités de médiation relèvent du comité de résidents, dont ils forment un sous-comité ${ }^{120}$. Halegua souligne que les comités de résidents fonctionnent comme des succursales des gouvernements locaux, en les aidant à mettre en œuvre les politiques et les programmes de même qu'en leur fournissant de l'information. Le comité de résidents dépend du gouvernement local pour son budget de fonctionnement et le salaire de ses membres ${ }^{121}$. Le règlement de 2002 sur la médiation populaire prévoit que les comités de médiation peuvent compter trois personnes ou plus, lesquelles peuvent être membres du comité de résidents, nommées par ce dernier ou élues par les résidents. Le directeur ou le secrétaire du Parti dirige le sous-comité de sécurité publique de la plupart des comités de résidents et, par le fait même, les comités de médiation ${ }^{122}$. En pratique, le travail de médiation revient aux membres du comité de résidents car ce sont eux qui, les premiers, entendent la demande de médiation des parties ou de l'une d'entre elles. Lubman souligne qu'avec les réformes, le nombre de membres du comité de médiation de même que leur qualité ont décliné. Plusieurs étaient, comme avant la Révolution culturelle, des femmes à la maison ou des travailleurs à la retraite. Par ailleurs, des cadres retraités sont aussi devenus médiateurs $^{123}$. Cependant, l'enthousiasme pour le travail de médiateur a considérablement diminué, notamment en raison du caractère insuffisant de la rémunération qui en a conduit plusieurs à trouver d'autres moyens pour produire des revenus $^{124}$.

Il existe un consensus chez les chercheurs chinois à l'effet que la médiation extrajudiciaire a connu un déclin constant entre 1978 et $2010^{125}$. Le PCC a décidé

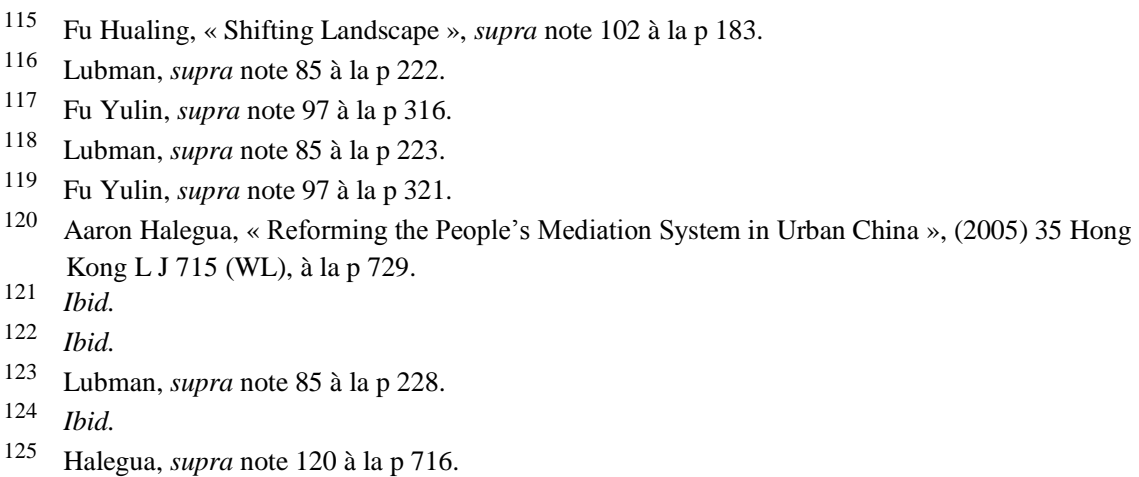


de tenter de revitaliser la médiation populaire et plusieurs instruments juridiques ont été promulgués à cette fin entre 1989 et 2010. Le professeur Song Wuchao a effectué une enquête de terrain sur la médiation extrajudiciaire, mais au début de la décennie 2000. Il signale tout d'abord que, dans la décennie 1990, la médiation populaire a perdu de sa crédibilité aux yeux des justiciables chinois ${ }^{126}$. La médiation n'apparaissait plus comme un moyen approprié de résoudre les conflits dans une société régie par l'État de droit socialiste. Song Wuchao avance plusieurs autres raisons de poids qui ont contribué au déclin de la médiation populaire. Au premier chef, il souligne la faible «qualité » des médiateurs ${ }^{127}$. Il ressort des résultats de son enquête que seulement 14 p. 100 des personnes agissant à titre de médiateurs populaires étaient titulaires, en 2008, d'un diplôme d'études secondaire ou plus élevé. C'est dire que 86 p. 100 des médiateurs populaires n'avaient pas terminé une formation de niveau secondaire ${ }^{128}$. Song Wuchao établit un lien entre le faible niveau d'éducation des médiateurs populaires et le financement très insuffisant des gouvernements locaux, dont relèvent les salaires des membres des comités de médiation populaire. Il a constaté lors de son enquête que les médiateurs populaires touchaient une compensation mensuelle allant de 20 à 60 yuans, c'est-à-dire de 4 à 10 dollars canadiens par mois ${ }^{129}$. Cependant, le tiers des médiateurs populaires ne recevaient aucune compensation financière ${ }^{130}$. Song Wuchao mentionne aussi un autre facteur, soit celui de l'absence de règles devant guider le processus de médiation. Ce dernier était trop flexible, dénué de balises concernant des points essentiels tels les suivants: le caractère public ou confidentiel de la médiation, la possibilité, pour les médiateurs, de convier des tiers au processus de médiation. Sur ce point, il n'est fait aucune référence à la nécessité de consulter les justiciables parties au processus ${ }^{131}$. De même, Song Wuchao souligne que les médiateurs populaires n'ont aucun moyen à leur disposition pour protéger les intérêts de la partie la plus vulnérable, ce qui peut, à terme, favoriser la partie la plus puissante ${ }^{132}$. En outre, le principe du caractère volontaire de la médiation a été bafoué par des comités de médiation populaire qui ont très fortement incité les justiciables en conflit à entamer le processus de médiation $^{133}$. De plus, alors que les cours populaires devraient soutenir et guider le travail des comités de médiation populaire, en réalité, très peu d'entre elles établissent des relations avec ces derniers ${ }^{134}$.

Plusieurs autres juristes chinois ont avancé des raisons complémentaires à la perte de crédibilité de la médiation populaire. Liu et Li soulignent que la pratique de la médiation durant la période maoïste, totalement régie par la lutte des classes et la «ligne des masses », surtout pendant la Révolution culturelle, a laissé un

126 Song Wuchao, supra note 47 à la p 102.

127 Ibid à la p 109. Les Chinois emploient en effet le terme « qualité », que nous appliquons en Occident aux objets, pour parler des personnes. Ces dernières sont de bonne ou de mauvaise «qualité ». 
mauvais souvenir aux justiciables chinois ${ }^{135}$. Zhu Jingwen cite deux facteurs expliquant pourquoi les justiciables ont peu à peu délaissé la médiation au profit de recours devant les tribunaux judiciaires. D'une part, le peu d'efficacité de la médiation $^{136}$ : les médiateurs ont traité, en comparaison des juges pour la même période, beaucoup moins de cas. D'autre part, Zhu Jingwen affirme que les médiateurs sont de plus en plus apparus comme des amateurs par rapport aux juges qui, eux, ont été engagés dans la voie de la professionnalisation ${ }^{137}$. Faisant écho à Song Wuchao, You Tiancheng, juge à la Cour populaire intermédiaire du Yunnan, explique que le travail de médiateur est devenu, au début des réformes, peu attrayant eu égard à la rémunération et aux conditions de travail. Il en est découlé un problème pour la rétention de médiateurs compétents ${ }^{138}$. Xu Xin souligne la complexité croissante des causes des conflits dans une société chinoise en mutation depuis $1978^{139}$. Or, les médiateurs n'avaient pas la formation nécessaire pour s'adapter à ces changements multiples et traiter les nouvelles causes de conflits. Xu Xin situe aussi le travail des médiateurs de l'époque dans son contexte. Le plus souvent, les médiateurs habitaient le même quartier que les parties au processus, ce qui a ouvert la porte à des atteintes à l'intégrité de leur travail en raison de l'influence des relations (guanxi) ${ }^{140}$. Les méthodes de travail des médiateurs ont souvent reposé selon Xu Xin sur l'imposition d'une logique d'harmonie aux parties sans tenir compte du tout des faits ni des lois et règlements applicables. Ils ont régulièrement mal différencié ce qui était illégal par rapport à ce qui était légal. Tous ces facteurs ont contribué à entamer sérieusement la crédibilité de la médiation extrajudiciaire, car les résultats du processus satisfaisaient de moins en moins les justiciables ${ }^{141}$. Néanmoins, les autorités judiciaires veulent contrer ce déclin, ce qui a conduit à l'élaboration puis à l'entrée en vigueur de la Loi sur la médiation.

\section{LA MÉDIATION POPULAIRE EN CHINE : UN FRAGILE MOYEN D'ACCÈS À LA JUSTICE.}

La Loi sur la médiation a été promulguée le 28 août 2010 et est en vigueur depuis le $1^{\mathrm{er}}$ janvier 2011. Au moment de la mise à jour du présent texte à des fins de publication, il n'existe aucune étude sur la pratique de la médiation sous ce nouveau régime et les cas traités par les commissions de médiation ne sont pas non plus accessibles, pour un ensemble de raisons ${ }^{142}$. Quelques observations

135 Liu et Li, supra note 93 à la p 256.

136 Jingwen Zhu, « Trends and Retrospection of Legalization: Analysis on the Data of Legislation and Litigations in China », (2010) 5:2 Front Law China 215, à la p 203 [Zhu, « Trends »].

137 Ibid.

138 You Tiancheng, « Renmin Tiaojie yao de ming, jue de kai, zou de yuan » [La médiation populaire : absolument...], au para 3 ; en ligne : Dongfangfayan $<$ http://www.d-f-f-y.com/faxueti/ss/201012/-2010-1212-114847.htm>, 12 décembre 2010.

139 Xu Xin, «Wanshan Renmin Tiaojie Zhidu yu Goujian Hexie Shehui » [Améliorer la médiation populaire et construire la société harmonieuse], para 5 ; en ligne : Civillaw.com.cn <http: //www.civillaw.com.cn/article/ default.asp?id=5017>, 13 septembre 2009.

$140 \quad$ Ibid au para 6.

141 Ibid.

142 Les vérifications ont été effectuées auprès de juristes chinois et de la banque de données Chinalawinfo. Les cas traités par les commissions de médiation ne sont pas tous consignés par 
préliminaires peuvent être formulées, en prenant appui sur les travaux visant la période précédente et le texte de la nouvelle loi.

La médiation populaire instaurée par la loi est définie comme «le processus par lequel les commissions populaires de médiation vont persuader les parties à un conflit de conclure un accord de médiation fondé sur les principes d'égalité des parties dans la négociation et sur leur consentement libre » ${ }^{143}$. Le PCC est obnubilé par la crainte des conflits sociaux et le risque qu'ils dégénèrent en mouvements de contestations de plus grande ampleur. Aussi n'est-il pas étonnant de trouver la mention, à l'article premier de cette loi, qu'elle a pour objectifs de résoudre les conflits en temps utile et de maintenir l'harmonie sociale et la stabilité ${ }^{144}$.

\section{A. La Loi sur la médiation}

Les commissions populaires de médiation [CPM], principal acteur institutionnel dans le processus de médiation, sont, dans le cadre de leur travail, assujetties, dans l'ordre : au respect des principes d'égalité des parties et de leur libre consentement, au respect des lois, règlements et politiques de l'État, de même qu'au respect des droits des parties. Cette dernière exigence implique que les commissions de médiation s'abstiennent de tenter d'empêcher les parties de faire valoir leurs droits par des recours en arbitrage, des recours administratifs ou des recours judiciaires ${ }^{145}$. Les parties peuvent choisir les médiateurs qui traiteront leur dossier ou encore, accepter le choix des médiateurs qui leur sera proposé par la commission populaire de médiation ${ }^{146}$. Cette dernière est définie comme étant une « organisation de masse » formée légalement afin de régler les conflits survenant entre les masses populaires ${ }^{147}$. La loi distingue deux intensités d'obligations en ce qui a trait à la création des commissions de médiation : elle est obligatoire pour les comités de villageois et les comités de quartier, mais laissée à la discrétion des entreprises et d'autres institutions publiques ${ }^{148}$. Les commissions comprennent entre trois et neuf membres, dont le mandat est de trois ans et est renouvelable une fois ${ }^{149}$. Les membres des commissions, soit les médiateurs populaires, sont élus ${ }^{150}$. Dans le cas des commissions de médiation dans le monde rural, les médiateurs populaires sont élus soit par l'assemblée des villageois, soit par l'assemblée des représentants du village ou par l'assemblée des résidents (du village). Les entreprises ou autres institutions ont la faculté de créer ou non des commissions populaires de médiation. Si elles le font, les membres de ces commissions sont élus, selon le cas, par l'assemblée des employés (en l'absence de syndicat), par les représentants des employés ou par le syndicat. Une fois constituées, les

écrit. En outre, même en présence d'un accord écrit, ces documents ne sont pas accessibles au public.

Loi sur la médiation, supra note 7 art 2.

144 Fan, «Jiufen Jiejue », supra note 72 à la p 471, affirme que la fonction de résolution des conflits est la première en importance assignée à la médiation populaire. Les citations sont notre traduction.

145 Ibid art 3.

146 Ibid par 23(1).

147 Ibid art 7.

148 Ibid art 8

149 Ibid art 9

150 Ibid art 9. Les précisions qui suivent sont tirées du même article. 
commissions de médiation doivent élaborer les règles applicables à leur travail de médiation. Également, elles sont tenues d'être à l'écoute des opinions du peuple et d'accepter la supervision de leur travail de médiation par le peuple ${ }^{151}$. Par ailleurs, les tribunaux populaires de base ont aussi un droit de regard sur le travail des commissions de médiation puisqu'ils sont, aux termes de l'article 5, chargés de guider leur travail.

$\mathrm{La}$ loi comprend quelques indications relatives au bagage attendu des médiateurs populaires. L'article 14 stipule qu'ils doivent être des citoyens adultes, impartiaux, décents, dévoués au travail de médiation populaire, posséder un certain niveau d'éducation et une certaine compréhension des politiques (de l'État) de même que des connaissances en droit. Les bureaux de la justice à l'échelon du district dispenseront une formation aux médiateurs sur une base régulière ${ }^{152}$. Le médiateur est assujetti à certaines obligations qui sont formulées suivant une gradation dans les sanctions pour violation de celles-ci ${ }^{153}$. Ainsi, les actes prohibés consistent à faire preuve de favoritisme envers une partie ${ }^{154}$, insulter une partie, exiger de l'argent, des biens ou autre avantage illicite et, enfin, divulguer des éléments tenant à la vie privée des parties ou leurs secrets commerciaux. Les sanctions vont de la réprimande à la perte du statut de médiateur, en passant par la critique et l'éducation du médiateur. Toutefois, nous faisons face à un silence de la loi pour ce qui est de la relation entre les actes prohibés et les sanctions. L'absence de données sur le fonctionnement du nouveau régime ne nous permet pas d'établir avec certitude les liens des médiateurs avec le PCC. Chose certaine, aux termes de l'article 5 de la Loi, les bureaux locaux du ministère de la Justice, pour les échelons du district et au-dessus, sont censés « guider » le travail des commissions de médiation et donc, des médiateurs.

La crainte que les conflits finissent par prendre une grande ampleur ressort clairement de l'article 21 où l'objectif de les régler en temps utile est à nouveau énoncé en relation avec la nécessité d'empêcher qu'ils s'intensifient. Cette possibilité d'aggravation du conflit est par ailleurs clairement prévue à l'article 25 . En vertu de cet article, les médiateurs populaires sont tenus de prendre des précautions supplémentaires à cet égard et de signaler aux organes locaux de sécurité publique le risque d'escalade et de transformation du conflit en matière pénale.

Le nouveau régime prévoit la gratuité de la médiation pour les parties. Les commissions populaires de médiation se voient en effet interdire de facturer le moindre coût pour leur travail de médiation ${ }^{155}$. Le financement de la médiation populaire incombe aux gouvernements locaux aux niveaux du district et aux échelons supérieurs ${ }^{156}$. Plus précisément, les fonds nécessaires doivent être fournis par les comités de villageois, les comités de quartier, les entreprises et les institutions publiques ${ }^{157}$.

151 Ibid art 11.

152 Ibid art 14

153 Ibid art 15.

154 Le terme chinois désigne le favoritisme et non pas la partialité, qui, elle, peut jouer en faveur d'une partie ou contre elle.

155 Ibid art 4.

156 Ibid art 6.

157 Ibid art 12. 
La médiation se déroule en plusieurs volets. Le premier concerne la décision de soumettre le cas à la commission populaire de médiation. En vertu de l'article 17, les parties disposent d'un droit de saisine direct, mais la commission de médiation peut aussi proposer, sur une base volontaire, de traiter le cas. Le même article précise que la médiation ne peut avoir lieu si une partie a expressément refusé de résoudre le conflit par la médiation. Les tribunaux populaires de base et les organes locaux de sécurité publique peuvent aussi faire savoir aux parties qu'elles peuvent saisir la commission de médiation ${ }^{158}$. Selon la nature du conflit, les parties, ou la commission de médiation, désigneront un ou plusieurs médiateurs pour traiter le cas ${ }^{159}$. Une fois la médiation commencée, le médiateur populaire dispose d'un éventail de moyens pour mener à bien son travail. Le recours à ces moyens est assujetti à l'approbation préalable des parties ${ }^{160}$. S'il l'estime nécessaire, le médiateur peut inviter des membres de la famille, des voisins, des collègues de travail, des personnes possédant des connaissances spécialisées et des personnes membres des organisations sociales pertinentes à participer au processus. Des balises sont énoncées au deuxième paragraphe de l'article 20 concernant les personnes appelées à participer au processus : elles doivent être impartiales, décentes, dévouées au travail de médiation et leur participation doit être approuvée par la population locale ${ }^{161}$. En ce qui concerne les normes applicables, les médiateurs, dans le cadre du processus, ont l'obligation de s'en tenir aux principes, d'effectuer des raisonnements juridiques et de rendre justice aux parties $^{162}$. Des précisions s'imposent ici. Tout d'abord, les principes dont il est question ne se limitent pas aux principes juridiques. En effet, ni dans la version originale en chinois ni dans la traduction officielle en anglais le mot «juridique » n'est juxtaposé au mot « principe ». Que faut-il entendre alors ? Il importe aussi de signaler que la traduction officielle en anglais ne reprend pas complètement l'article 21. En effet, un élément important du répertoire normatif des médiateurs ne figure pas dans la traduction anglaise, soit les gongde. Ce terme signifie l'éthique de la société qui a cours à une période précise. Or, cette omission n'est pas sans lien avec la question des principes. À la lumière du régime qui a prévalu avant la réforme de 2010, où les médiateurs ont recouru aux qingli, ceux-ci faisant partie des gongde ${ }^{163}$, il est loisible de supposer que les médiateurs pourront mobiliser un autre répertoire normatif que les lois et règlements. Cependant, dans les discours officiels sur la nouvelle loi, l'accent est mis sur le fait que la médiation est fondée d'abord sur les lois et règlements. Les propos du juge You Tiancheng appuient notre interprétation. You Tiancheng affirme clairement que la médiation doit d'abord être fondée sur les règles de droit étatiques (yifa tiaojie), mais de manière non exclusive. Les médiateurs doivent aussi tenir compte des relations entre les parties et de leurs sentiments respectifs, et travailler à un règlement du conflit qui reçoive l'assentiment de la société. Tout cela est inclus

158 Ibid art 18.

159 Ibid art 22.

160 Ibid art 20.

161 Les exigences énoncées à ce paragraphe recoupent en partie celles qui sont imposées aux médiateurs, avec la différence qu'ici, l'approbation de la population locale est requise pour que la personne participe à la médiation.

162 Ibid art 21. La Loi ne précise pas de quels principes il s'agit.

163 Liu et Li, « Minjian », supra note 93 aux pp 259-260. 
dans la notion de "gongde ». L'omission, dans la traduction officielle anglaise, de mentionner les gongde est-elle due à un simple oubli ou est-elle délibérée ? À l'heure actuelle, il est impossible de trancher.

Un aperçu des divers volets du travail attendu des médiateurs populaires ressort de l'article 22. Outre l'obligation d'entendre les dépositions des parties, ils doivent expliquer les lois, les règlements et les politiques qui s'appliquent. Ils doivent user de patience pour être persuasifs et proposer des solutions fondées sur les principes d'égalité des parties dans la négociation et la compréhension mutuelle. Enfin, les médiateurs doivent aider les parties à conclure un accord de médiation sur la base du libre consentement. La loi laisse entendre que l'exercice du métier de médiateur peut parfois être périlleux. En effet, l'article 16 stipule qu'un médiateur blessé ou devenu handicapé dans l'exercice de ses fonctions a le droit de recevoir une aide médicale et une allocation de la part du gouvernement local.

De leur côté, les parties ont des droits et des obligations. Au chapitre des droits, elles peuvent choisir les médiateurs qui traiteront leur cas ou accepter ceux qui leur sont proposés. Elles peuvent accepter ou refuser de se soumettre à la médiation et, si elles ont consenti au processus, demander en tout temps qu'il y soit mis fin. La médiation peut, selon leur choix, se dérouler en privé ou en public. Finalement, les parties peuvent exprimer librement leur volonté et parvenir à un accord sur la base du consentement libre ${ }^{164}$. Cela dit, les parties doivent exposer sans mentir les faits qui donnent lieu au conflit, respecter l'ordre sur les lieux de la médiation et témoigner du respect aux médiateurs. Elles doivent aussi respecter l'exercice des droits de chacune d'elles ${ }^{165}$.

Le processus de médiation se solde de l'une ou l'autre des manières suivantes : ou il échoue, ou il réussit. En cas d'échec, les médiateurs mettent fín à la médiation et informent les parties des autres moyens dont elles disposent pour faire valoir leurs droits, à savoir l'arbitrage, les recours administratifs ou les recours judiciaires. Si les parties parviennent à un accord, ce dernier peut être écrit $^{166}$ ou verbal ${ }^{167}$. Dans ce dernier cas, le contenu de l'accord est consigné par les médiateurs. Le travail de transcription de l'accord est plus important si ce dernier revêt une forme écrite. Il contient alors les renseignements sur les parties, les faits importants du conflit, la source précise du conflit, la responsabilité de chacune des parties, le contenu de l'accord et ses modalités d'exécution. L'accord prend effet le jour où il porte la signature des parties, celle des médiateurs et le sceau de la commission populaire de médiation ${ }^{168}$. Il revêt une force contraignante pour les parties $^{169}$. Si nécessaire, les tribunaux judiciaires (ici les tribunaux populaires de base), dûment saisis par les parties dans les 30 jours de la date d'entrée en vigueur de l'accord, peuvent en confirmer la force exécutoire. Cette confirmation a pour effet de conférer à une partie le droit de s'adresser au tribunal pour obtenir l'exécution de l'accord dans le cas où l'autre partie ne donne pas suite aux termes de l'accord. Les commissions populaires de médiation sont appelées, quoique de

164 Loi sur la médiation, supra note 7 art 23.

165 Ibid art 24.

166 Ibid art 29.

167 Ibid art 30.

168 Ibid.

169 Ibid art 31. 
manière assez vague, à favoriser la mise en œuvre de l'accord intervenu entre les parties en exerçant un certain pouvoir de «supervision » ${ }^{170}$. Advenant un différend entre les parties relativement à l'exécution de l'accord de médiation, elles peuvent saisir un tribunal judiciaire ${ }^{171}$.

\section{B. Des problèmes structurels persistants}

Les données dont nous disposons nous portent à penser que la mise en œuvre du régime de médiation populaire fera face à divers obstacles. Les juristes chinois ont mis en lumière les problèmes qui minaient le régime jusqu'à la promulgation de la Loi sur la médiation. Ces problèmes, nombreux, ont été exposés dans la partie qui précède. Il s'agit à présent de déterminer si la Loi sur la médiation apporte des solutions. Notre réponse est plutôt négative.

En effet, plusieurs des problèmes qui pesaient sur le régime précédent demeurent. Tout d'abord, aucune solution n'est apportée au financement inadéquat du régime. Le financement relève toujours des gouvernements locaux. Compte tenu des échelons administratifs et territoriaux énumérés dans la Loi sur la médiation, le nouveau régime de médiation extrajudiciaire est destiné au monde rural plutôt qu'au monde urbain. Or, depuis le milieu de la décennie 1990, la Chine a amorcé un processus de décentralisation qui se traduit par le transfert, aux divers échelons administratifs sous le gouvernement central, de plusieurs chefs de dépenses $^{172}$. Ce contexte défavorise grandement les échelons administratifs du monde rural, soit les districts, les cantons et les villages dont plus de la moitié connaissent de très graves difficultés financières ${ }^{173}$. Dans cette perspective, le fait que le financement du nouveau régime de médiation extrajudiciaire soit confié aux gouvernements locaux est susceptible de compromettre sa mise sur pied, du moins dans le cas des échelons administratifs les plus pauvres.

La persistance du problème de la rémunération adéquate des médiateurs populaires risque à son tour de perpétuer le problème lié au recrutement de médiateurs compétents par les commissions populaires de médiation. Même si la Loi sur la médiation augmente substantiellement les exigences en ce qui a trait aux compétences des médiateurs, il est loin d'être dit que les personnes répondant, le cas échéant, aux qualifications requises, choisissent d'agir à titre de médiateurs.

Aussi, même en supposant que des commissions populaires de médiation soient établies dans le monde rural, la question de la crédibilité de la médiation aux yeux des justiciables reste entière.

Un trait propre au modèle chinois ressort du nouveau régime. Comme à l'époque maoïste, les médiateurs sont tenus d'expliquer non seulement le droit applicable mais aussi les politiques du $\mathrm{PCC}^{174}$. Ce travail est difficilement conciliable avec la neutralité attendue des médiateurs car ils ont en quelque sorte un point de vue à défendre. D'autres remarques s'imposent relativement au processus. En vertu de la Loi sur la médiation, le processus de médiation populaire

70 Ibid

171 Ibid art 32.

172 Tao Chiu Lam, « The county system and county governance », dans Jae Ho Chung et Tao Chiu

173 Ibid.

Lam (dir.), China's Local Administration, Routledge, New York, 2010, 149, aux pp 166-167.

174 Lubman, supra note 85 à la p 59. 
est gratuit pour les parties. La gratuité joue en faveur de l'accès à la justice mais cet accès est selon nous miné par un ensemble de facteurs.

Sous le régime de la nouvelle loi, l'intégrité du processus de médiation risque de subir des atteintes importantes en raison de l'intervention possible des organes de sécurité publique dans les cas jugés délicats. L'intérêt des organes de sécurité publique réside dans le maintien de la stabilité sociale et leur logique est basée sur la répression des conflits. Or, l'intérêt des parties exige que les médiateurs puissent travailler à une véritable résolution du conflit qui les oppose plutôt qu'à sa suppression pure et simple. L'on ne saurait sous-estimer l'importance que revêt pour le régime le maintien de la stabilité sociale. Haitian Lu affirme que la part du budget qui est consacrée aux activités «weiwen » (protéger la stabilité) est presque aussi élevée que celle qui est consacrée à la défense nationale ${ }^{175}$. Dans ce contexte, il est fort à craindre que les médiateurs et les parties subissent une logique d'harmonie imposée, contraire aux objectifs qui sous-tendent la mise sur pied du nouveau régime. Le climat actuel n'est pas sans rappeler celui qui prévalait dans la Chine maoïste, période durant laquelle le régime visait à éradiquer les conflits plutôt qu'à traiter ses causes véritables ${ }^{176}$.

Une autre source d'interférence possible avec le travail des commissions populaires de médiation réside dans le fait que celles-ci doivent être «à l'écoute du peuple». Cette petite mention, apparemment anodine, dans la Loi sur la médiation, mérite qu'on s'y arrête. Elle inquiète. Outre l'atteinte au caractère confidentiel du processus, elle pourrait permettre de livrer une des parties à la vindicte d'une ou plusieurs personnes, et la loi ne prévoit aucun mécanisme de gestion de cette situation. Plusieurs auteurs ont exprimé l'opinion que l'ensemble de l'appareil judiciaire chinois opère dans un climat de populisme qui constitue une source de pression pour toutes les professions juridiques ${ }^{177}$. En effet, l'orientation prônée par l'actuel président de la CPS, Wang Shengjun, consiste en une justice, rendue par les divers organes judiciaires, qui tient compte de l'opinion publique $^{178}$. Cette justice doit être «pour le peuple» et le modèle de juge mis de l'avant est celui d'un «juge du peuple». Or, la nouvelle Loi sur la médiation reflète cette tendance au populisme judiciaire. Nul ne sait dans quelle direction peuvent aller les commentaires du peuple sur le travail des commissions de médiation, ni la suite qu'elles doivent, le cas échéant, y donner. Fan Yu apporte sur ce point un éclairage contestable selon nous. Elle affirme que la participation des masses donne au processus un caractère ouvert et démocratique ${ }^{179}$. Chose certaine, les commissions populaires de médiation subiront des pressions. Or, que vaut une solution proposée aux parties sur la base de «l'opinion populaire»? Quels sont les fondements de sa légitimité ? Par qui doit-elle être déterminée ?

175 Haitian Lu, « State Channelling of Social Grievances : Theory and Evidence in China », (2011)

176 41 Hong Kong Law Journal 547 (WL), à la p 549.

176 Ibid.

177 Zang Dongsheng, « Rise of Political Populism and the Trouble with the Legal Profession in China » (2010) $6: 1$, Harvard China Review 79, à la p 93 ; en ligne : SSRN <http://ssrn.com/abstract=1585442> ; Zhang, supra note 14 aux pp 256-257 ; Minzner, supra note 10 à la $\mathrm{p} 6$.

178 Zhang, supra note 14 aux pp 256-257.

179 Fan Yu, «Zhonghua Renmin Gongheguo Renmin Tiaojie Fa Pingxi » [Une analyse de la Loi de République populaire de Chine sur la médiation], (2011) 2 Faxuejia 1, à la p 6. 
Bien que la Loi sur la médiation fasse une large place au droit étatique comme fondement du processus, l'absence de mesures tangibles visant la formation des médiateurs suggère que ceux-ci continuent de recourir majoritairement aux qingli, ou gongde, soit le sens de la justice d'une population précise dans un contexte bien délimité. Or, ce sens de la justice des communautés villageoises est susceptible d'entrer en contradiction directe avec le droit édicté par l'État et de promouvoir des solutions parfois discriminatoires contre l'une des parties. Pour sa part, Zhu Suli favorise largement le recours, par les médiateurs, à d'autres normes que la règle de droit. Pour lui, le fait de fonder la médiation extrajudiciaire sur les règles de droit relève de l'antinomie ${ }^{180}$. Par ailleurs, si la médiation extrajudiciaire s'éloigne des règles de droit, il n'en résulte pas selon lui que la société perçoit le processus et le résultat comme injustes ${ }^{181}$. Fan $\mathrm{Yu}$ abonde dans son sens en critiquant l'orientation, selon elle trop légaliste de la loi ${ }^{182}$. À l'instar de Zhu Suli, elle estime que la médiation ne fait pas appel à la compétence professionnelle mais bien à l'expérience et au savoir-faire du médiateur, lesquels ne s'apprennent pas dans une faculté de droit ${ }^{183}$. Elle est ouvertement en faveur des gongde qui possèdent, comme composantes du répertoire normatif des médiateurs, une valeur découlant du fait qu'ils ne sont pas des règles de droit. Exiger que les gongde n'aillent pas à l'encontre du droit étatique constitue pour elle une erreur ${ }^{184}$. C'est toute la question de la normativité du monde rural qui est en cause ici.

Bien que loi interdise expressément aux commissions populaires de médiation de tenter d'empêcher les justiciables d'aller devant les tribunaux, cette protection est hautement fragilisée par le contexte politique. En effet, les autorités judiciaires estiment que les recours devant les tribunaux exacerbent les conflits au lieu de les résoudre $^{185}$. Aussi, la réponse appropriée consiste-t-elle à détourner les justiciables des tribunaux, jugés trop contentieux, pour les canaliser vers la médiation extrajudiciaire, censée permettre une résolution harmonieuse des conflits ${ }^{186}$. Dans cette perspective, il est possible que des parties subissent des pressions visant à les empêcher de saisir les tribunaux locaux, tout comme les personnes voulant user du système des pétitions font l'objet de moyens d'intimidation de la part des autorités ${ }^{187}$.

D'autres questions se posent. Les commissions populaires sont-elles assujetties à des quotas de causes à régler ? Comme les tribunaux judiciaires le $\operatorname{sont}^{188}$, la

80 Zhu, « Tiaojie », supra note 43 à la p 12.

181 Ibid à la p 13.

182 Fan Yu, supra note 179 à la p 5.

183 Ibid à la $\mathrm{p} 11$.

184 Ibid à la p 5.

185 Leila Choukroune et Antoine Garapon, « Les normes de la société harmonieuse », (2007) 3

Perspectives Chinoises 38. C'est la position des autorités judiciaires telle qu'elle est rapportée par les auteurs à la $\mathrm{p} 40$. Cela est toujours vrai aujourd'hui, comme l'indiquent les articles du China Daily, journal qui livre le point de vue officiel. Wang Huazhong, « Mediation, not trials, can help settle rising disputes »; en ligne : <www.china-daily.com.cn/cndy/201006/29/content_10031749.htm>.

186 Leila Choukroune et Antoine Garapon, supra note 185 à la p 48.

187 Zhang Qianfan, « Performance or Petitions », Caixin ; en ligne : <http://english.caing.com/201010-20/100190626.html>.

188 Minzner, supra note 10 à la p 38. 
possibilité que les commissions le soient aussi est réelle. Si tel est le cas, ce qui signifie qu'elles pourraient être à la recherche de conflits à régler, cette contrainte pourrait les conduire, comme ce fut le cas par le passé, à intervenir directement auprès de justiciables avant même que ceux-ci décident de recourir à la médiation $^{189}$. Cette situation minerait totalement le caractère volontaire de la médiation.

\section{CONCLUSION}

L'état des lieux, en ce qui concerne l'accès à la justice dans la Chine des réformes, est quelque peu problématique. Tout d'abord, le système judiciaire étatique touche des limites relativement à sa capacité de traiter le nombre de causes qui lui sont soumises. Par conséquent, le fait, pour le PCC, de se tourner vers la médiation populaire semble logique si l'on conçoit la médiation extrajudiciaire comme un moyen d'accès à la justice. Comme ce fut le cas en Occident, et notamment en Amérique du Nord, il était possible d'envisager un système judiciaire aux multiples visages, où les nouveaux modes de règlement des conflits, dont la médiation extrajudiciaire, opèrent en complément des tribunaux étatiques. Il était de prime abord tentant d'attribuer au renouveau de la médiation populaire en Chine les mêmes motivations que celles qui ont présidé à son essor en Occident. Cependant, cette analyse ne tient que très partiellement la route. Le contexte chinois colore fortement la nature de la médiation qui y est pratiquée, de même que ses finalités.

Le souci de désengorger les tribunaux par l'entremise de la médiation est effectivement une préoccupation du PCC et des autorités judiciaires chinoises. Ce point de vue est validé par Fan Yu. Elle détaille un contexte qu'elle appelle la crise de la justice civile en Chine, où les tribunaux judiciaires ne fournissent tout simplement plus à la tâche en raison de l'augmentation constante du nombre de causes $^{190}$. Les limites du système judiciaire étatique seraient selon elle à la source $\mathrm{du}$ renouveau de la médiation extrajudiciaire en Chine ${ }^{191}$. Cependant, si, en Occident, et particulièrement aux États-Unis, le recours à la médiation extrajudiciaire a été justifié par des considérations d'accès à la justice, il en va autrement en Chine. L'accès à la justice n'est qu'une considération très secondaire aux yeux du régime, obnubilé par le maintien de la stabilité sociale. Le régime assigne à la médiation extrajudiciaire l'objectif d'assurer l'harmonie de la société chinoise dans son entier. Les conflits, dans la Chine des réformes, sont perçus par le PCC comme une menace pour la stabilité sociale, mais aussi politique dans la mesure où ils mettent en cause, à terme, la survie même du régime qui est resté très nerveux depuis le massacre de la place Tian An Men en $1989^{192}$. Cette crainte politique des dirigeants chinois distingue la trajectoire chinoise vers la médiation extrajudiciaire. La société des réformes est tout sauf une société harmonieuse. Elle

\footnotetext{
189 Stanley Lubman avait relevé cette tendance pour la période maoïste. Lubman, supra note 85 à la $\mathrm{p}$ 48.

190 Fan, « Jiufen Jiejue », supra note 72 à la p 380.

191 Ibid.

192 Yan Zhong, « Chinese township government: between a rock and a hard place », dans Jae Ho Chung et Tao-Chiu Law, dir., China's Local Administration. Tradition and changes in the subnational hierarchy, Londres et New York, Routledge, 2010, 175, à la p 186.
} 
est en proie à une myriade de conflits sociaux qui prennent diverses formes et dont le nombre va en augmentant ${ }^{193}$. Dans cette perspective, le mot d'ordre de la société harmonieuse assujettit tout l'appareil d'État, dont le système judiciaire, à l'objectif du maintien de la stabilité sociale. En ce sens, la pression sur les tribunaux judiciaires est bien réelle ${ }^{194}$.

Les justiciables chinois sont les premiers concernés par cette nouvelle orientation du régime. Pendant des années, ce dernier les a incités à saisir les tribunaux judiciaires et à user du droit comme d'une arme. Les justiciables chinois ont saisi cette possibilité et abondamment usé des tribunaux judiciaires, délaissant la médiation populaire. Le PCC doit maintenant infléchir cette tendance qu'il a luimême initiée, et ce n'est pas chose facile compte tenu de l'évolution du rapport au droit des justiciables chinois depuis le début des réformes. La rhétorique de l'harmonie est destinée d'abord aux justiciables chinois, qui sont priés de bien vouloir se conformer à la représentation qui en est faite.

L'appel à une tradition reconstruite constitue un élément problématique du contexte chinois de la médiation extrajudiciaire. La démarche n'est pas propre à la Chine cependant. Ainsi, l'Inde, peu de temps après son indépendance, a aussi présenté la médiation comme une ressource endogène à valoriser face aux modèles juridiques importés de l'Occident ${ }^{195}$.

En outre, il existe une similarité entre le cas indien et le cas chinois : le renouveau de la médiation extrajudiciaire est en réalité une initiative de l'État, mais il est présenté comme une ressource endogène, recourant ainsi à la tradition réinventée ${ }^{196}$. En présentant la médiation comme paradigme local, le PCC ajoute une dimension fortement nationaliste à celle-ci. Le nationalisme résonne très fort dans la société chinoise surtout quand, comme dans le cas présent, il s'en prend à l'Occident. Cette connotation endogène dont est parée la médiation lui confère une légitimité difficilement attaquable. Cependant, en Chine, l'accent mis sur la médiation populaire n'a pas toujours reposé sur un appel à l'harmonie. La période maoïste a été régie par le mot d'ordre de la lutte des classes, et le conflit était perçu comme un obstacle au développement du socialisme ${ }^{197}$. Or, si la rhétorique du régime marque un changement radical de paradigme depuis la période maoïste, passant de la lutte des classes à la société harmonieuse, un point commun unit ces deux moments de l'histoire chinoise. Il s'agit non pas de résoudre le conflit pour le mieux-être des parties, suivant une logique de médiation transformatrice par exemple, mais de le dissoudre purement et simplement ${ }^{198}$. Cet objectif, qui s'explique dans le contexte chinois, annihile cependant les avantages de la médiation extrajudiciaire telle qu'elle est conçue dans la doctrine nord-américaine, où la valeur de la responsabilisation des parties (empowerment) occupe une place importante. Le processus de médiation populaire est «sous influence». Sa finalité

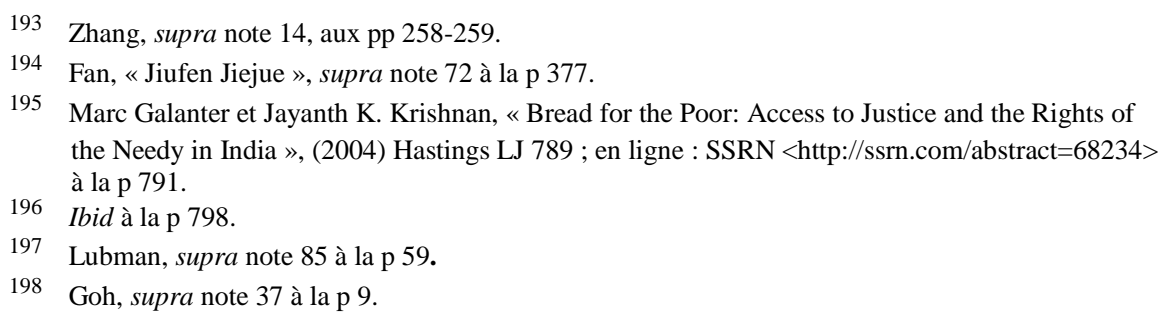


de contrôle social débouche sur une logique d'harmonie imposée, contraire aux intérêts des parties à un conflit.

Il se pourrait que le PCC soit sur un terrain glissant, car le régime de médiation populaire instauré par la Loi sur la médiation est trop compromis par divers facteurs - exposés dans le présent texte - pour pouvoir répondre aux aspirations, bien réelles, de la population chinoise en matière de justice. Aussi longtemps que la médiation populaire en Chine ne sera pas conçue comme un véritable mode de résolution des conflits, fondée sur le libre consentement des parties et facilitée par un tiers neutre, les justiciables chinois y trouveront peu d'attrait et de pertinence. Qu'adviendra-t-il de ces conflits, peut-être soumis aux comités de médiation populaire, mais non traités sur le fond? Le recours aux tribunaux étatiques ne pourra davantage répondre aux attentes des justiciables chinois. En effet, les tribunaux étatiques subissent aussi l'emprise de la médiation, judiciaire cette fois, comme paradigme local avec variation sur thème ${ }^{199}$. Si les médiateurs populaires et les juges sont contraints de jouer le jeu du paradigme local réinventé afin de satisfaire les autorités, rien ne dit que les justiciables chinois, pour leur part, seront prêts à le faire. L'histoire récente de la Chine témoigne de sa capacité à s'engager en profondeur dans des réformes. Il faut espérer que ce «virage contre le droit» dont parle Minzner ne soit que d'une durée temporaire ${ }^{200}$ et que les réformes juridiques prennent une direction plus favorable à l'ensemble de la société chinoise.

199 Stanley Lubman, « Civil Litigation Being Quietly 'Harmonized' », en ligne: <http://blogs.wsj.com/china-realtime/2011/05/31/civil-litigation-being-quietlyharmonized/tab/print/>. Dans ce texte, Stanley Lubman expose la pression des autorités judiciaires en faveur de la médiation judiciaire.

Minzner, supra note 10. 